OPEN ACCESS

Check for updates

\section{Risks of ischaemic heart disease and stroke in meat eaters, fish eaters, and vegetarians over 18 years of follow-up: results from the prospective EPIC-Oxford study}

\author{
Tammy Y N Tong, ${ }^{1}$ Paul N Appleby, ${ }^{1}$ Kathryn E Bradbury, ${ }^{1}$ Aurora Perez-Cornago, ${ }^{1}$ Ruth C Travis, ${ }^{1}$ \\ Robert Clarke, ${ }^{2}$ Timothy J Key ${ }^{1}$
}

${ }^{1}$ Cancer Epidemiology Unit,

Nuffield Department of

Population Health, University of

Oxford, Richard Doll Building,

Oxford OX3 7LF, UK

${ }^{2}$ Clinical Trial Service Unit and Epidemiological Studies Unit, Nuffield Department of

Population Health, Big Data Institute, University of Oxford, Oxford, UK

Correspondence to: TY N Tong tammy.tong@ndph.ox.ac.uk (or @tammy_tong on Twitter ORCID 0000-0002-0284-8959)

Additional material is published online only. To view please visit the journal online.

Cite this as: BMJ 2019;366:14897 http://dx.doi.org/10.1136/bmj.l4897

Accepted: 10 July 2019

\section{ABSTRACT}

OBJECTIVE

To examine the associations of vegetarianism with risks of ischaemic heart disease and stroke.

DESIGN

Prospective cohort study.

SETTING

The EPIC-Oxford study, a cohort in the United Kingdom with a large proportion of non-meat eaters, recruited across the country between 1993 and 2001.

\section{PARTICIPANTS}

48188 participants with no history of ischaemic heart disease, stroke, or angina (or cardiovascular disease) were classified into three distinct diet groups: meat eaters (participants who consumed meat, regardless of whether they consumed fish, dairy, or eggs; $n=24$ 428), fish eaters (consumed fish but no meat; $n=7506)$, and vegetarians including vegans $(n=16$ 254), based on dietary information collected at baseline, and subsequently around $2010(n=28$ 364).

MAIN OUTCOME MEASURES

Incident cases of ischaemic heart disease and stroke (including ischaemic and haemorrhagic types) identified through record linkage until 2016.

RESULTS

Over 18.1 years of follow-up, 2820 cases of ischaemic heart disease and 1072 cases of total stroke (519 ischaemic stroke and 300 haemorrhagic stroke) were recorded. After adjusting for sociodemographic and lifestyle confounders, fish eaters and vegetarians had $13 \%$ (hazard ratio $0.87,95 \%$ confidence interval 0.77 to 0.99$)$ and $22 \%(0.78,0.70$ to 0.87$)$

\section{WHAT IS ALREADY KNOWN ON THIS TOPIC}

Vegetarian and vegan diets have become increasingly popular in recent years, but the potential benefits and hazards of these diets are not fully understood Previous studies of two diet groups have reported that vegetarians have lower risks of ischaemic heart disease than non-vegetarians

However, no evidence has been reported of a difference in the risk of mortality from stroke, possibly because of limited available data and lack of available evidence on stroke subtypes

\section{WHAT THIS STUDY ADDS}

This study showed that fish eaters and vegetarians (including vegans) had lower risks of ischaemic heart disease than meat eaters

Vegetarians (including vegans) had higher risks of haemorrhagic and total stroke than meat eaters

Further research is needed to replicate these results in other populations and to identify mediators that might contribute to the observed associations lower rates of ischaemic heart disease than meat eaters, respectively ( $\mathrm{P}<0.001$ for heterogeneity). This difference was equivalent to 10 fewer cases of ischaemic heart disease (95\% confidence interval 6.7 to 13.1 fewer) in vegetarians than in meat eaters per 1000 population over 10 years. The associations for ischaemic heart disease were partly attenuated after adjustment for self reported high blood cholesterol, high blood pressure, diabetes, and body mass index (hazard ratio $0.90,95 \%$ confidence interval 0.81 to 1.00 in vegetarians with all adjustments). By contrast, vegetarians had $20 \%$ higher rates of total stroke (hazard ratio 1.20, 95\% confidence interval 1.02 to 1.40 ) than meat eaters, equivalent to three more cases of total stroke ( $95 \%$ confidence interval 0.8 to 5.4 more) per 1000 population over 10 years, mostly due to a higher rate of haemorrhagic stroke. The associations for stroke did not attenuate after further adjustment of disease risk factors.

\section{CONCLUSIONS}

In this prospective cohort in the UK, fish eaters and vegetarians had lower rates of ischaemic heart disease than meat eaters, although vegetarians had higher rates of haemorrhagic and total stroke.

\section{Introduction}

Vegetarian and vegan diets have become increasingly popular in recent years, partly due to perceived health benefits, as well as concerns about the environment and animal welfare. ${ }^{1}$ In the United Kingdom, both the representative National Diet and Nutrition Survey 2008-12 and a 2016 Ipsos MORI survey estimated about 1.7 million vegetarians and vegans living in the country. $^{2}{ }^{3}$ Evidence suggests that vegetarians might have different disease risks compared with nonvegetarians, ${ }^{4}$ but data from large scale prospective studies are limited, because few studies have recruited sufficient numbers of vegetarian participants.

For ischaemic heart disease, some but not all previous studies reported significantly lower risks of mortality from ischaemic heart disease in vegetarians than in non-vegetarians. ${ }^{5-7}$ In terms of incidence, the only previous study (the European Prospective Investigation into Cancer (EPIC)-Oxford) reported that vegetarians had a lower risk of ischaemic heart disease than non-vegetarians, ${ }^{8}$ but at the time of publication the study had an insufficient duration of follow-up to separately examine the risks in other diet groups (fish eaters and vegans).

For stroke, two previous reports, ${ }^{5}{ }^{6}$ including one that included EPIC-Oxford data, ${ }^{6}$ found no 
significant differences in risk of total stroke deaths between vegetarians and non-vegetarians. However, no previous studies have examined the incidence of stroke in relation to vegetarian diets, or have examined the main stroke types.

We report here the risks of both incident ischaemic heart disease and stroke in people with distinct dietary habits-that is, meat eaters, fish eaters and vegetarians (including vegans)-with a separate evaluation of ischaemic and haemorrhagic strokes, over 18 years of follow-up in the EPIC-Oxford study.

\section{Methods}

\section{Study population and design}

EPIC-Oxford is a prospective cohort study of about 65 000 men and women who were recruited across the UK between 1993 and 2001. Details of the recruitment process have been described previously. ${ }^{9}$ Individuals were recruited from either general practices or by postal questionnaire. The general practice recruitment method recruited 7421 men and women aged 35 to 59 who were registered with participating general practices, all of whom completed a full questionnaire on their diet, lifestyle, health characteristics, and medical history. The postal recruitment preferentially targeted vegetarians, vegans, and other people interested in diet and health, and recruited 57990 participants aged 20 or older. A full questionnaire was mailed to all members of the Vegetarian Society and all surviving participants of the Oxford Vegetarian Study, ${ }^{10}$ and respondents were invited to provide names and addresses of relatives and friends who were also interested in receiving a questionnaire. A short questionnaire was also distributed to all members of the Vegan Society, enclosed in vegetarian and health food magazines, and displayed in health food shops; and a full questionnaire was subsequently mailed to all those who returned the short questionnaire.

Despite the targeted recruitment of the postal method, about $80 \%$ of meat eaters in the cohort were recruited by post. Subsequently, a follow-up questionnaire was sent to participants in 2010, which asked similar questions on their diet and lifestyle, and participants returned the questionnaires between 2010 and 2013. A participant flowchart of the recruitment process and inclusion into this study is shown as supplementary figure 1 . The study protocol was approved by a multicentre research ethics committee (Scotland A Research Ethics Committee) and all participants provided written informed consent.

\section{Assessment of diet group and diet}

The full baseline questionnaire collected responses to four questions about consumption of meat, fish, dairy products, and eggs, in the form of "Do you eat any meat (including bacon, ham, poultry, game, meat pies, sausages)?" or similar for the other three food groups. These four questions were used to classify participants into meat eaters (participants who reported eating meat, regardless of whether they ate fish, dairy, or eggs), fish eaters (participants who did not eat meat but did eat fish), vegetarians (participants who did not eat meat or fish, but did eat one or both of dairy products and eggs), and vegans (participants who did not eat meat, fish, dairy products, or eggs). The follow-up questionnaire sent in 2010 included identical questions on consumption of meat, fish, dairy products, and eggs (yes/no). Therefore, at both baseline and follow-up, participants were classified into one of four diet groups: meat eaters, fish eaters, vegetarians, and vegans. Owing to the small number of vegans, vegetarians and vegans were combined as one diet group in the main analyses, but the two groups were examined separately for each outcome in secondary analyses.

The baseline questionnaire also included a semiquantitative food frequency section containing 130 items, which asked about dietary intake over the past year, and which was previously validated using 16 days (in four sets of four days) of weighed dietary records and selected recovery and concentration biomarkers. ${ }^{11-13}$ For calculation of food and nutrient intakes, the frequency of consumption of each food or beverage was multiplied by a standard portion size (mostly based on data from the UK Ministry of Agriculture, Fisheries, and Food $)^{14}$ and nutrient content of each food or beverage (based on McCance and Widdowson's food composition tables). ${ }^{15}$ Because our prespecified analysis plan was to examine disease risks associated with distinct dietary groups, the associations of individual foods and nutrients with risks were not assessed in this study, but information on intakes of foods and nutrients were used in descriptive and secondary analyses.

\section{Assessment of other characteristics}

In addition to diet, the baseline questionnaire also asked questions on sociodemographic characteristics, lifestyle, and medical history, including questions on education level, smoking, physical activity, use of dietary supplements, and use of oral contraceptives or hormone replacement therapy in women. Socioeconomic status was categorised by use of the Townsend deprivation index, ${ }^{16}$ based on the participants' postcodes. For physical activity, based on their responses to questions asked about their occupation and their time spent participating in activities including walking, cycling, and other physical exercises, participants were categorised by a validated physical activity index with four levels. ${ }^{17}$ Alcohol consumption was determined from responses to five items on the food frequency questionnaire. Questions relating to smoking and alcohol consumption were also asked on the follow-up questionnaire in 2010.

For biological measurements, body mass index was calculated from participants' self reported height and weight at recruitment, which was previously found to be accurate compared with measured height and weight in a validation study of about 4800 participants. ${ }^{18}$ All participants were also asked at recruitment whether they were willing to have their blood pressure measured at their general practice and 
to provide a blood sample. Details of the procedures for blood pressure measurement and blood sample collection, which were conducted in subsets of the cohort, have been previously reported. ${ }^{81920}$

\section{Outcome ascertainment}

Participants were followed up via record linkage to records from the UK's health service up to 31 March 2016. Outcomes of interest were ischaemic heart disease (codes 410-414 from ICD-9 (international classification of diseases, 9th revision) or codes I20-I25 from ICD-10), including acute myocardial infarction (ICD-9 410 or ICD-10 I21); and total stroke (ICD-9 430-431, 433-434, 436; or ICD-10 I60-I61, I63-I64), including ischaemic stroke (ICD-9 433-434 or ICD-10 I63) and haemorrhagic stroke (ICD-9 430-431 or ICD10 I60-I61). Details of events, using the relevant ICD-9 or ICD-10 codes, were obtained from hospital records or death certificates.

\section{Exclusion criteria}

Participants who were not resident in England, Wales, or Scotland ( $\mathrm{n}=945)$ were excluded, as were those with no Hospital Episode Statistics data or NHS number $(n=20)$. We also excluded participants who completed the short questionnaire only $(n=7619)$; were younger than $20(n=1)$ or older than 90 at recruitment $(n=58)$; had no follow-up (were censored at or before the date of recruitment (eg, if they were living abroad), $n=364$ ); could not be traced by the NHS $(n=14)$; had an unknown diet group (if they did not answer the relevant questions to be classified, $n=132$ ); had unreliable nutrient data ( $\geq 20 \%$ of food frequencies missing, or daily energy intakes $<500 \mathrm{kcal}$ or $>3500 \mathrm{kcal}$ for women or $<800$ $\mathrm{kcal}$ or $>4000 \mathrm{kcal}$ for men $(1 \mathrm{kcal}=4.18 \mathrm{~kJ}=0.00418$ MJ), $n=1219)$; had a self reported history of acute myocardial infarction, stroke, or angina at recruitment $(n=6837)$; or had a date of diagnosis that preceded or equalled the date of recruitment $(n=14)$.

\section{Statistical analyses}

Baseline characteristics and food and nutrient intakes of the EPIC-Oxford participants were summarised by diet group. For self reported body mass index, and measures of blood pressure (systolic and diastolic blood pressure) and blood lipids (total cholesterol, high density lipoprotein cholesterol (HDL-C), nonHDL-C), the means and 95\% confidence intervals are presented, after adjustment for sex and age at entry (in 5-year age groups), alcohol consumption ( $<1,1-7$, $8-15, \geq 16 \mathrm{~g} /$ day), and physical activity (inactive, low activity, moderately active, very active, unknown). ${ }^{17}$

Cox proportional hazards regression models were used to estimate the hazard ratios and 95\% confidence intervals for the associations between diet group (meat eaters, fish eaters, vegetarians including vegans) and each outcome of interest, with meat eaters as the reference group. For participants who completed both the baseline and follow-up questionnaire, diet group and relevant time varying covariates (smoking and alcohol consumption) were updated at follow-up. The underlying time variable was the age at recruitment to the age at diagnosis, death, or administrative censoring, whichever occurred first. For acute myocardial infarction or ischaemic heart disease, events were censored on the respective outcomes of interest. For total stroke, ischaemic stroke, and haemorrhagic stroke, events were censored on any stroke. All analyses were stratified by sex, method of recruitment (general practice or postal), and region (seven regions across the UK), and adjusted for year of recruitment (per year from 1994 to 1999), education (no qualifications, basic secondary (eg, O level), higher secondary (eg, A level), degree, unknown), Townsend deprivation index (quarters, unknown), ${ }^{16}$ smoking (never, former, light, heavy, unknown), alcohol consumption ( $<1,1-7,8-15, \geq 16 \mathrm{~g} /$ day), physical activity (inactive, low activity, moderately active, very active, unknown), dietary supplement use (no, yes, unknown), and oral contraceptive use (no, yes ever, unknown) and hormone replacement therapy use (no, yes ever, unknown) in women.

We used Wald tests to test for heterogeneity of risk between diet groups. The proportional hazards assumption was assessed on the basis of Schoenfeld residuals, and was not violated for the variables of interest in the adjusted model for either ischaemic heart disease or stroke ( $\mathrm{P}>0.05$ for all categories). Self reported history of high blood pressure (no, yes, unknown), high blood cholesterol (no, yes, unknown), diabetes (no, yes, unknown), and body mass index $(<20,20-22.5,22.5-25,25-27.5, \geq 27.5$, unknown) were assessed as potential physiological mediators, since these factors were known to be associated with vegetarian diets, ${ }^{19-23}$ as well as being established cardiovascular risk factors. ${ }^{24}$ Total fruit and vegetable intake, total dietary fibre, and total energy intake (each continuous) were assessed as possible relevant dietary factors. We assessed the effects of potential physiological mediators and possible relevant dietary factors by adding each variable one at a time to the previous model. An additional model was also fitted including all potential physiological mediators.

To estimate the population impact of vegetarian diets on cardiovascular health, we assessed the absolute risk difference for each outcome between meat eaters, fish eaters, and vegetarians. Predicted incidence and absolute risk differences were presented as per 1000 population over 10 years, and were estimated by use of hazard ratios and 95\% confidence intervals expressed as floating absolute risks, ${ }^{25}{ }^{26}$ which do not alter the value of the hazard ratios but assign an appropriate 95\% confidence interval to all groups, including the reference group (thereby allowing an estimation of the uncertainty in the effect size in the reference group). In meat eaters, predicted incidence over this time period of each outcome was calculated as (1$\left.\mathrm{S}_{\mathrm{r}}\right) \times 1000$, where $\mathrm{S}_{\mathrm{r}}=(1-$ observed incidence in meat eaters $)^{10}$, and represents the predicted 10 year survival (that is, non-incidence) in meat eaters. By subtracting this estimate of survival from 1 , and multiplying by 1000 , the resulting estimate represents incidence 
per 1000 population over 10 years. For all other diet groups, predicted incidence was calculated as (1$\left.\mathrm{S}_{\mathrm{r}}^{\mathrm{HR}}\right) \times 1000$, where $\mathrm{HR}$ represents the hazard ratio or confidence intervals for each outcome in that diet group. By applying HR or confidence interval estimates in each diet group as an exponential to survival in the reference group, $\mathrm{S}_{\mathrm{r}}{ }^{\mathrm{HR}}$ represents the predicted 10 year survival rate in the each of the other diet groups. Absolute risk differences were then calculated as the crude differences between the predicted incidence per 1000 population over 10 years between each diet group and the meat eaters.

Additional sensitivity analyses included analyses using baseline diet group only, excluding participants with less than five years of follow-up, including participants recruited via the postal method only, censoring at age 70 or setting entry time at age 70 to evaluate possible differences by age at event, and performing multiple imputation (with 10 imputations) for missing covariates. The percentages of missing values in the covariates were $12.7 \%$ for the Townsend deprivation index, $10.9 \%$ for physical activity, $6.3 \%$ for education level, and less than $2 \%$ for each of the other covariates. We assessed heterogeneity in the associations between diet group and risk of ischaemic heart disease or stroke by sex, age at recruitment ( $<60$ or $\geq 60$ years), smoking status (never, former, or current), body mass index ( $<25$ or $\geq 25$ ), presence of risk factors (one or more of self reported history of high blood pressure, high blood cholesterol, or diabetes), and any long term treatment for any illness or condition (no, yes) by adding appropriate interaction terms to the Cox models and testing for statistical significance of interaction across strata using likelihood ratio tests. All analyses were performed with Stata version 14.1 or 15.1 (Stata Corp, TX, United States) and P values less than 0.05 were considered significant.

\section{Patient and public involvement}

No members of the community or patients were involved in setting the research question or the outcome measures, nor were they involved in developing plans for recruitment, design, or implementation of the study. They were not asked to advise on interpretation or writing up of results. We are appreciative of our participants who, although not partners, were engaged in the progress of EPIC-Oxford through follow-up questionnaires. The results are disseminated to study participants through newsletters and the study website (www.epic-oxford.org/).

\section{Results}

\section{Cohort characteristics}

The analyses included 48188 participants, of whom 28364 had reported on diet both at baseline and at follow-up on average 14 years later. Of these participants with repeated measures of diet, 13972 (96\%) of 14540 meat eaters at baseline remained meat eaters at follow-up. Of fish eaters and vegetarians at baseline, 2608 (57\%) of 4555 and 6746 (73\%) of 9269 were in the same diet group at follow-up, respectively.
Cohort characteristics of the study participants for each of the three diet groups (meat eaters, fish eaters, vegetarians including vegans) at baseline are presented in table 1 (and separately for vegetarians and vegans in supplementary table 1). Overall, non-meat eaters were younger and had a lower area level socioeconomic status than meat eaters, but were more highly educated, less likely to smoke, reported slightly lower alcohol consumption, were more physically active, and were more likely to report dietary supplement use. They were also less likely to report previous high blood pressure, high blood cholesterol, or diabetes; or receive long term treatment for illnesses. In women, non-meat eaters were more likely to report oral contraceptive use but less likely to report use of hormone replacement therapy. Non-meat eaters reported having lower body mass index and had lower measured blood pressure than meat eaters. For blood lipids, vegetarians had about $0.5 \mathrm{mmol} / \mathrm{L}$ lower plasma concentrations of total cholesterol and non-HDL-C than meat eaters. Fish eaters had slightly higher plasma concentrations of HDL-C than meat eaters, while vegetarians had slightly lower concentrations.

Food and nutrient intakes in the different diet groups are presented in table 2 and supplementary table 2 (separately for vegetarians and vegans). Intakes of most foods and nutrients differed between the dietary groups: for example, vegetarians had higher intakes of fruit and vegetables, legumes and soya foods, nuts, and dietary fibre, and had a lower intake of saturated fat $(10 \% v 12 \%$ energy) and sodium than meat eaters.

\section{Association of diet groups with cardiovascular diseases}

Over 18.1 years of follow-up in 48188 participants, 2820 cases of ischaemic heart disease (including 788 cases of acute myocardial infarction) and 1072 cases of total stroke (including 519 cases of ischaemic stroke and 300 cases of haemorrhagic stroke) were reported. Fish eaters (hazard ratio 0.87, 95\% confidence interval 0.77 to 0.99$)$ and vegetarians $(0.78,0.70$ to 0.87 ) had lower rates of ischaemic heart disease than meat eaters $\left(\mathrm{P}_{\text {heterogeneity }}<0.001\right.$ between all diet groups; fig 1). Conversely, vegetarians, but not fish eaters, had significantly higher rates of haemorrhagic stroke than meat eaters $\left(1.43,1.08\right.$ to $\left.1.90 ; \mathrm{P}_{\text {heterogeneity }}=0.04\right)$ and higher rates of total stroke $(1.20,1.02$ to 1.40 ; $P_{\text {heterogeneity }}=0.06$ ). We saw no significant differences between diet groups for the risk of acute myocardial infarction or ischaemic stroke. When we assessed vegetarians and vegans separately, the point estimates for vegans were lower for ischaemic heart disease (0.82, 0.64 to 1.05$)$ and higher for total stroke (1.35, 0.95 to 1.92$)$ than meat eaters, but neither estimate was statistically significant, possibly because of the small number of cases in vegans, as indicated by the wide confidence intervals (supplementary table 3).

When the roles of potential mediators of the associations were assessed, the associations between diet group and ischaemic heart disease were marginally attenuated when adjusted for self reported history 


\begin{tabular}{|c|c|c|c|}
\hline \multirow{2}{*}{ Characteristics } & \multicolumn{3}{|l|}{ Diet group* } \\
\hline & Meat eaters $(n=24428)$ & Fish eaters $(n=7506)$ & Vegetarians $(n=16254)$ \\
\hline \multicolumn{4}{|l|}{ Sociodemographic characteristics } \\
\hline Age, years (mean (standard deviation)) & $49.0(13.1)$ & $42.1(12.8)$ & $39.4(13.1)$ \\
\hline Sex, women (number (\%)) & $18481(75.7)$ & $6186(82.4)$ & $12232(75.3)$ \\
\hline Top socioeconomic quarter (number (\%))† & $5959(28.0)$ & $1431(21.9)$ & $3018(21.2)$ \\
\hline Higher education (number (\%)) & $7374(32.8)$ & $3308(46.2)$ & $6698(43.3)$ \\
\hline \multicolumn{4}{|l|}{ Lifestyle } \\
\hline Current smokers (number (\%)) & $2955(12.1)$ & $764(10.2)$ & $1685(10.4)$ \\
\hline Alcohol consumption, g/day (mean (standard deviation)) & $10.1(12.9)$ & $10.0(12.3)$ & $9.3(12.8)$ \\
\hline Moderate/ high physical activity (number (\%)) & $6752(31.2)$ & $2684(40.2)$ & $5849(40.0)$ \\
\hline Dietary supplement use (number (\%)) & $13295(55.6)$ & $4702(64.1)$ & $8961(56.1)$ \\
\hline \multicolumn{4}{|l|}{ Medical history (number (\%)) } \\
\hline Prior high blood pressure & $2938(12.1)$ & $549(7.3)$ & $935(5.8)$ \\
\hline Prior high blood cholesterol & $1616(6.6)$ & $255(3.4)$ & $345(2.1)$ \\
\hline Prior diabetes & $353(1.4)$ & $61(0.8)$ & $93(0.6)$ \\
\hline Receiving long term treatment for any illness & $7022(29.1)$ & $1622(21.9)$ & $3077(19.1)$ \\
\hline Oral contraceptive use§ & $13263(72.2)$ & $4959(80.5)$ & $9620(79.0)$ \\
\hline Hormone replacement therapy use§ & $4484(24.6)$ & $728(11.9)$ & $954(7.9)$ \\
\hline \multicolumn{4}{|l|}{ Biological measurements (adjusted mean $(95 \% \mathrm{CI})) \uparrow$} \\
\hline Body mass index & $24.1(24.0$ to 24.1$)$ & 23.1 (23.0 to 23.2$)$ & $23.0(23.0$ to 23.1$)$ \\
\hline Systolic blood pressure $(\mathrm{mm} \mathrm{Hg})$ & $125.7(125.4$ to 126.1$)$ & $123.4(122.7$ to 124.2$)$ & $123.7(123.2$ to 124.2$)$ \\
\hline Diastolic blood pressure $(\mathrm{mm} \mathrm{Hg})$ & 77.1 (76.9 to 77.3$)$ & 75.5 (75.0 to 76.0$)$ & 75.9 (75.6 to 76.2$)$ \\
\hline Total cholesterol (mmol/L) & $5.50(5.46$ to 5.54$)$ & $5.31(5.23$ to 5.39$)$ & $4.98(4.92$ to 5.03$)$ \\
\hline HDL cholesterol (mmol/L) & $1.32(1.31$ to 1.33$)$ & $1.35(1.32$ to 1.38$)$ & $1.29(1.27$ to 1.31$)$ \\
\hline Non-HDL cholesterol (mmol/L) & 4.18 (4.14 to 4.22$)$ & $3.96(3.88$ to 4.04$)$ & $3.68(3.62$ to 3.74$)$ \\
\hline \multicolumn{4}{|c|}{$\begin{array}{l}\text { For high density lipoprotein }(\mathrm{HDL}) \text { cholesterol, P value for heterogeneity was } 0.002 \text { between diet groups, and less than } 0.001 \text { for all other variables. } \\
\text { *Meat eaters were participants who reported eating meat, regardless of whether they ate fish, dairy, or eggs; fish eaters were participants who did not eat } \\
\text { meat but did eat fish; vegetarians included vegans. } \\
\text { †Based on Townsend index. } \\
\text { fDefined as regularly taking any vitamins, minerals, fish oils, fibre, or other food supplements during the past } 12 \text { months. } \\
\text { §ln women only. } \\
\text { IBody mass index was based on self reported measures in the whole cohort. Blood pressure was measured in } 8862 \text { meat eaters, } 1742 \text { fish eaters, and } \\
4364 \text { vegetarians and vegans. Blood lipids were measured in } 1985 \text { meat eaters, } 566 \text { fish eaters, and } 1109 \text { vegetarians and vegans. Estimates were } \\
\text { adjusted for the cross stratification of sex and age at entry ( } 5 \text {-year age groups), alcohol consumption ( }(1,1-7,8-15, \geq 16 \mathrm{~g} / \text { day), and physical activity } \\
\text { (inactive, low activity, moderately active, very active, unknown). }\end{array}$} \\
\hline
\end{tabular}

\begin{tabular}{|c|c|c|c|}
\hline \multirow[b]{2}{*}{ Foods or nutrients } & \multicolumn{3}{|l|}{ Diet group* } \\
\hline & $\begin{array}{l}\text { Meat eaters } \\
(n=24 \text { 428) }\end{array}$ & $\begin{array}{l}\text { Fish eaters } \\
(n=7506)\end{array}$ & $\begin{array}{l}\text { Vegetarians* } \\
(\mathrm{n}=16254)\end{array}$ \\
\hline \multicolumn{4}{|l|}{ Foods } \\
\hline Total meat and meat products (g/day) & $76.2(48.5)$ & - & - \\
\hline Red and processed meat (g/day) & $50.3(38.9)$ & - & - \\
\hline Poultry (g/day) & $26.0(21.7)$ & - & - \\
\hline Total fish (g/day) & $41.9(29.1)$ & $38.5(33.3)$ & - \\
\hline Dairy milk (mL/day) & $324.2(184.7)$ & $274.0(189.8)$ & $232.3(207.3)$ \\
\hline Soya milk (mL/day) & $5.9(43.2)$ & $21.1(79.4)$ & $54.5(127.9)$ \\
\hline Dairy cheese (g/day) & $20.7(18.6)$ & $27.5(24.2)$ & $26.8(25.4)$ \\
\hline Total fresh fruit (g/day) & $264.8(207.5)$ & $291.1(228.0)$ & $283.8(239.2)$ \\
\hline Total vegetables (g/day) & $252.3(131.8)$ & $287.3(148.7)$ & $294.4(163.2)$ \\
\hline Legumes and soya foods (g/day) & $28.9(30.3)$ & $58.1(44.4)$ & $74.4(58.1)$ \\
\hline Nuts and nut butter (g/day) & $4.8(9.3)$ & $8.0(12.1)$ & $10.6(16.2)$ \\
\hline \multicolumn{4}{|l|}{ Nutrients } \\
\hline Carbohydrates (\% energy) & $48.0(6.2)$ & $51.0(6.5)$ & $52.8(6.8)$ \\
\hline Protein (\% energy) & $16.9(3.0)$ & $14.6(2.3)$ & $13.6(2.1)$ \\
\hline Total fat (\% energy) & $31.6(5.9)$ & $30.8(6.3)$ & $30.2(6.6)$ \\
\hline Saturated fat (\% energy) & $11.5(3.3)$ & $10.6(3.3)$ & $10.2(3.5)$ \\
\hline Monounsaturated fat (\% energy) & $10.7(2.3)$ & $9.9(2.4)$ & $9.7(2.6)$ \\
\hline Polyunsaturated fat (\% energy) & $6.3(1.9)$ & $7.0(2.2)$ & $7.1(2.5)$ \\
\hline Dietary fibre (g/day) & $18.8(6.7)$ & $21.2(7.4)$ & $22.1(8.0)$ \\
\hline Sodium (mg/day) & $2773(864)$ & $2684(864)$ & $2664(885)$ \\
\hline Total energy (kJ/day) & $8298(2250)$ & 7939 (2199) & $7813(2234)$ \\
\hline
\end{tabular}

Data are mean (standard deviation). For all variables, $\mathrm{P}$ values for heterogeneity was less than 0.001 between diet groups.

*Meat eaters were participants who reported eating meat, regardless of whether they ate fish, dairy, or eggs; fish eaters were participants who did not eat meat but did eat fish; and vegetarians included vegans. of high blood pressure, high blood cholesterol, and diabetes, and for self reported body mass index. The lower rate of ischaemic heart disease in vegetarians was of borderline significance when adjusted for all potential mediators simultaneously $(0.90,0.81$ to 1.00; supplementary table 4). For stroke, adjustments for potential mediators caused small changes in the hazard ratios; the association became slightly stronger for vegetarians after adjustment for self reported history of high blood pressure, and to a lesser extent when adjusted for history of diabetes or for body mass index. Additional adjustment for fruit and vegetable intake, total fibre, or total energy had little effect on the results.

\section{Absolute risk difference for vascular disease outcomes by diet group}

Table 3 shows the absolute risks of ischaemic heart disease and total stroke in the three diet groups. By comparing the absolute risk difference of each outcome by diet group, for an individual of the cohort's average age (44.7 years) and other characteristics (supplementary table 1), and not accounting for competing risk from other diseases or influences from other external factors, vegetarian diets were associated with 10 fewer (95\% confidence interval 6.7 to 13.1 fewer) cases of ischaemic heart disease per 1000 population over 10 years than meat eaters. Conversely, 


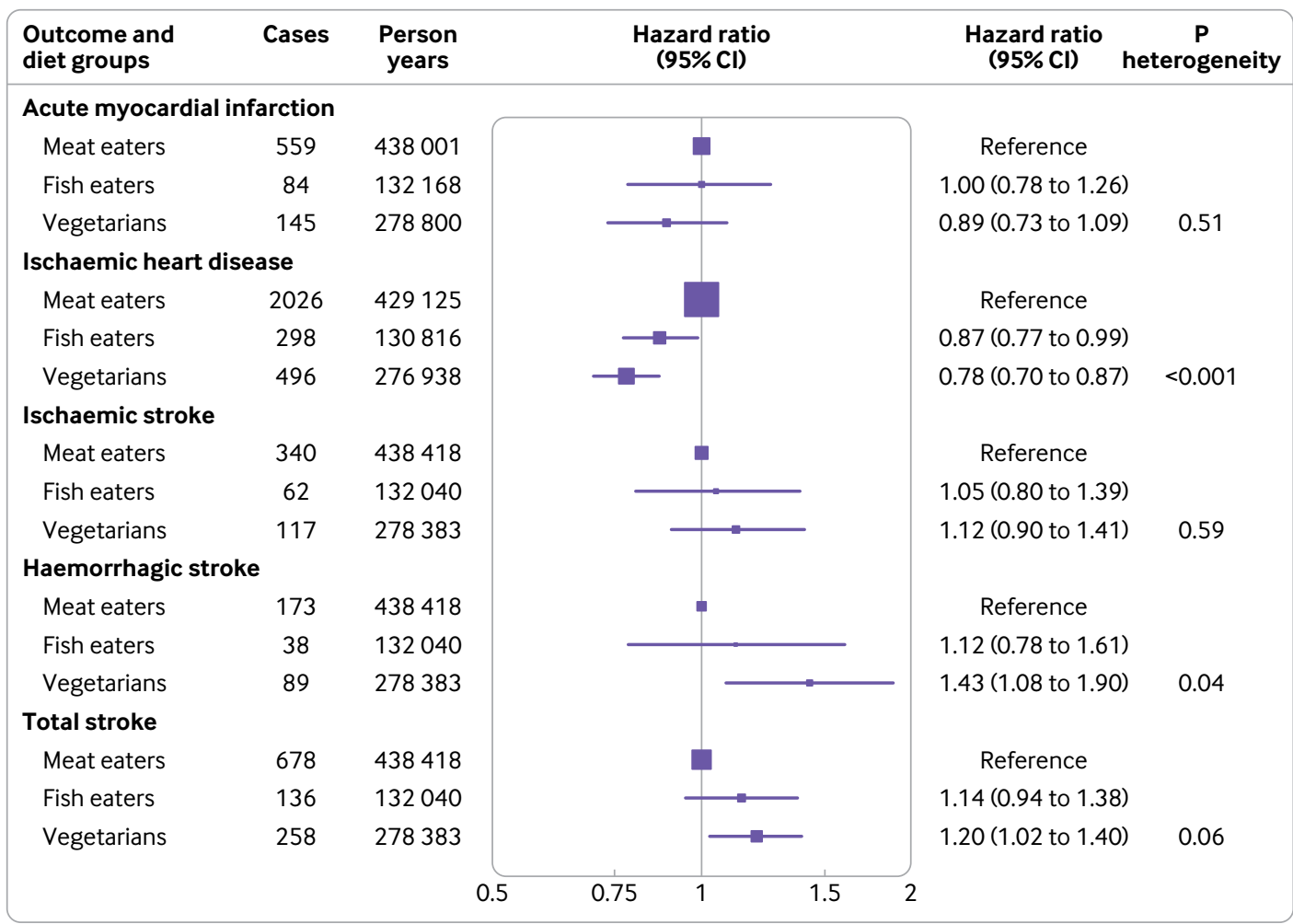

Fig 1 | Rates of ischaemic heart disease and stroke in fish eaters and vegetarians (including vegans) compared with meat eaters in the EPIC-Oxford study $(n=48188)$. Meat eaters were participants who reported eating meat, regardless of whether they ate fish, dairy, or eggs; fish eaters were participants who did not eat meat but did eat fish; and vegetarians included vegans. Meat eaters were used as the reference group throughout. All analyses included age as the underlying time variable; were stratified by sex, method of recruitment (general practice or postal), and region (seven categories); and were adjusted for year of recruitment (per year), education (no qualifications, basic secondary (eg, 0 level), higher secondary (eg, A level), degree, unknown), Townsend deprivation index (quarters, unknown), smoking (never, former, light, heavy, unknown), alcohol consumption ( $(1,1-7,8-15, \geq 16 \mathrm{~g} /$ day), physical activity (inactive, low activity, moderately active, very active, unknown), dietary supplement use (no, yes, unknown), and oral contraceptive and hormone replacement therapy use in women. $P$ heterogeneity=significance of heterogeneity in risk between diet groups based on Wald tests. Box sizes are proportional to the number of cases in each group

vegetarian diets were associated with three more $(95 \%$ confidence interval 0.8 to 5.4 ) cases of total stroke.

\section{Sensitivity and subgroup analyses}

In sensitivity analyses using baseline diet group only, excluding participants with less than five years

\begin{tabular}{|c|c|c|}
\hline Outcome and diet groups* & $\begin{array}{l}\text { Predicted incidence per } 1000 \\
\text { population over } 10 \text { yearst }\end{array}$ & $\begin{array}{l}\text { Absolute risk difference per } 1000 \\
\text { population over } 10 \text { years } \neq\end{array}$ \\
\hline \multicolumn{3}{|l|}{ Ischaemic heart disease } \\
\hline Meat eaters & 46.2 (43.8 to 48.7$)$ & Reference \\
\hline Fish eaters & 40.4 (36.2 to 45.2$)$ & $-5.8(-10.0$ to -1.0$)$ \\
\hline Vegetarians & 36.2 (33.1 to 39.5$)$ & $-10.0(-13.1$ to -6.7$)$ \\
\hline \multicolumn{3}{|l|}{ Total stroke } \\
\hline Meat eaters & 15.4 (14.1 to 16.8$)$ & Reference \\
\hline Fish eaters & 17.5 (14.8 to 20.6$)$ & $2.1(-0.6$ to 5.3$)$ \\
\hline Vegetarians & 18.3 (16.2 to 20.8$)$ & $3.0(0.8$ to 5.4$)$ \\
\hline \multicolumn{3}{|c|}{ 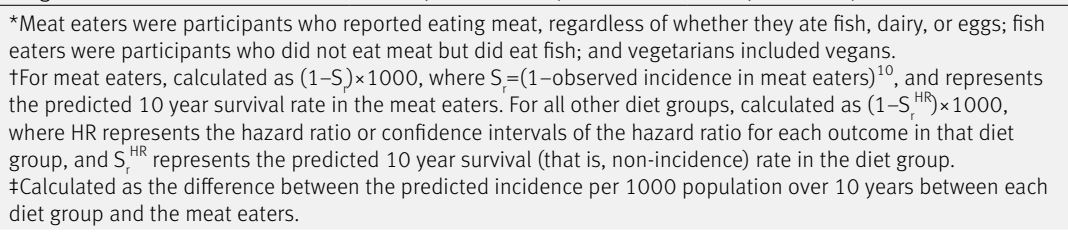 } \\
\hline
\end{tabular}

of follow-up, including participants recruited via the postal method only, and performing multiple imputations for missing covariates, the results were similar (supplementary table 5). When the analyses were censored at age 70 or limited to those above age 70 (setting entry time at age 70 ) respectively, a significantly higher rate of haemorrhagic stroke was observed in vegetarians who had an event above the age of 70 , but not in those who had an event before age 70 or were censored at that age. In subgroup analyses, significantly lower rates of ischaemic heart disease were observed among fish eaters only in those aged less than 60 years at recruitment, but lower rates were observed in vegetarians in both age groups (supplementary table 6). No other significant interactions by subgroups were observed for the association between diet group and ischaemic heart disease or stroke (supplementary tables 6-7).

\section{Discussion}

The present study examines the risks of both ischaemic heart disease and stroke, including subtypes, in meat eaters, fish eaters, vegetarians, and vegans in a cohort with a large proportion of non-meat eaters. 
We observed lower rates of ischaemic heart disease in fish eaters and vegetarians than in meat eaters, which appears to be at least partly due to lower body mass index and lower rates of high blood pressure, high blood cholesterol, diabetes associated with these diets. Conversely, vegetarians had higher risks of haemorrhagic and total stroke.

\section{Comparison with other studies}

In a collaborative meta-analysis of five previous prospective studies (Adventist Mortality Study, Health Food Shoppers Study, Adventist Health Study, Heidelberg Study, Oxford Vegetarian Study) with median recruitment between 1960 and 1980, vegetarians had a $24 \%$ lower rate ratio of death from ischaemic heart disease $(0.76,95 \%$ confidence interval 0.62 to 0.94 ) than non-vegetarians. ${ }^{5}$ In other analyses of death from ischaemic heart disease, the death rate ratios comparing vegetarians with nonvegetarians were $0.99(0.79$ to 1.23$)$ in a combined analysis of EPIC-Oxford and the Oxford Vegetarian Study, ${ }^{6} 0.70$ (0.41 to 1.18 ) in the German Vegetarian Study, ${ }^{27}$ and 0.81 (0.64 to 1.02) in the Adventist Health Study $2 .^{7}$ In a recent meta-analysis that pooled the estimates from these previous prospective studies, including a previous report from the EPIC-Oxford study that only compared vegetarians with non-vegetarians (meat and fish eaters combined), ${ }^{8}$ the risk ratio for ischaemic heart disease comparing vegetarians with non-vegetarians was 0.75 (0.68 to 0.82$).^{28}$

For stroke, previous analyses, including one study that included EPIC-Oxford data but with fewer cases than the present study, ${ }^{6}$ reported no significant differences in stroke mortality by diet group, ${ }^{56}$ and pooled analyses showed a similar result. ${ }^{28}$ However, previous studies only reported on stroke mortality, ${ }^{56}$ which might be strongly influenced by treatment as well as the underlying disease risk, ${ }^{28}$ and no studies were found which reported on the two subtypes of stroke.

\section{Interpretation of results and implications}

The results of the present study showed that fish eaters and vegetarians had lower risks of ischaemic heart disease than meat eaters. The associations were attenuated after adjustment for self reported high blood pressure, high blood cholesterol, diabetes, and body mass index, which suggests that part of the associations might be attributed to these factors. However, the lower risk in vegetarians and vegans remained marginally significant after adjustment for all of these factors. The reason for such differences is not certain, but could be partly attributed to lower concentrations of low density lipoprotein cholesterol (LDL-C; or non-HDL-C concentrations as a surrogate) associated with meatfree diets, ${ }^{29-31}$ differences that were not fully accounted for by adjusting for self reported high blood cholesterol. Previous meta-analyses of prospective studies showed that lower concentrations of non-HDL-C or LDL-C were associated with lower risks of ischaemic heart disease. ${ }^{26}{ }^{32}$ Furthermore, both randomised trials of statin treatments ${ }^{33}$ and mendelian randomisation studies $^{30}$ have confirmed a causal association of LDL-C with ischaemic heart disease. Hence, fish eaters and vegetarians, who have lower non-HDL-C (table 1) or LDL-C than meat eaters, ${ }^{19}{ }^{34}$ could have lower risks of ischaemic heart disease. However, the possible beneficial roles of generally healthier diets that included high intakes of fruit and vegetables, ${ }^{35}$ legumes, ${ }^{36}$ or fibre ${ }^{37}$ cannot be excluded, despite little change in the hazard ratios after adjustment for these individual components.

For stroke outcomes, the combined results from two randomised statin trials reported 21\% higher risks of haemorrhagic stroke per $1 \mathrm{mmol} / \mathrm{L}$ reduction in LDL-C, ${ }^{29}{ }^{38}$ which are consistent with results of observational studies of cholesterol concentrations and haemorrhagic stroke. ${ }^{39-41}$ A recent study that included observational and genetic evidence from China alongside the trial evidence from western countries has suggested this inverse association between LDL-C and haemorrhagic stroke might be causal. ${ }^{42}$ These previous studies corroborate the findings of the present study, indicating that vegetarians, who have relatively low LDL-C, had higher risks of haemorrhagic stroke. For ischaemic stroke, previous prospective studies and randomised trials have consistently shown weak positive associations of non-HDL-C or LDL-C levels with risk, ${ }^{26323338}$ which is supported by recent mendelian randomisation studies. ${ }^{31} 42$ This apparent discordance between previous evidence and those of the present study for LDL-C levels and ischaemic stroke suggests that other dietary factors associated with the lack of animal food consumption could contribute to the observed associations.

Results of several studies in Japan, showing that individuals with a very low intake of animal products had an increased incidence and mortality from haemorrhagic and total stroke, and also a possibly higher risk of ischaemic stroke mortality, ${ }^{43-46}$ suggest that some factors associated with animal food consumption might be protective for stroke. Vegetarians and vegans in the EPIC-Oxford cohort have lower circulating levels of several nutrients (eg, vitamin $\mathrm{B}_{12},{ }^{47}$ vitamin $\mathrm{D},{ }^{48}$ essential amino acids, ${ }^{49}$ and long chain n-3 polyunsaturated fatty acids ${ }^{50}$ ), and differences in some of these nutritional factors could contribute to the observed associations. ${ }^{45}$ 51-54 Serum concentrations of these nutritional factors and nonHDL-C have only been measured in a subset of the EPICOxford cohort, and therefore their role in the observed associations of vegetarian diets with ischaemic heart disease or stroke cannot be accurately determined in the current context, but should be further investigated.

High blood pressure is an established major risk factor for both ischaemic heart disease and stroke, ${ }^{24} 55$ and recent evidence confirmed this is true for all age groups, especially for haemorrhagic stroke. ${ }^{56}$ However, given that the vegetarians in the EPIC-Oxford study had lower blood pressure than the meat eaters, and the fact that the association between diet group and stroke risk became slightly stronger after adjustment for history of high 
blood pressure, this factor is unlikely to account for the higher risks of haemorrhagic and total stroke observed among vegetarians in the present study. Furthermore, although differential treatment of cardiovascular risk factors including hypertension in the different diet groups could influence their subsequent disease risk, this does not appear to be the case in the EPIC-Oxford study; previous analyses in this cohort have shown that although vegetarians had lower use of drug treatment overall than non-vegetarians, no significant differences were seen in the use of specific treatments for high blood pressure, high blood cholesterol, or diabetes by diet group, among those individuals who reported a diagnosis of these conditions. ${ }^{57}$

Misclassification of stroke cases, such as the misclassification of haemorrhagic stroke as ischaemic stroke, cannot be excluded as a possible explanation for the possibly discrepant results for ischaemic stroke, although previous adjudication studies of stroke types confirmed the reliability of hospital admission records and death certificates in the UK for diagnosis of stroke types in the UK population over this calendar period. ${ }^{5859}$ Although age is also an important risk factor for both ischaemic heart disease and stroke, and meat eaters in the present study were on average 10 years older than the vegetarians, age is known accurately in our cohort, and therefore any potential confounding or cohort effect is accounted for by the analyses that use age as the underlying time variable and further adjust for calendar year of entry.

The current study focused on the examination of risks associated with predefined dietary groups, and therefore the relative contributions of individual foods have not been assessed. Future research could benefit from dose-response analyses of foods that distinguish the diet groups, including meat, fish, dairy, and eggs, to identify possible optimal levels of consumption for balancing risks from different outcomes. Such analyses might be better performed in other large scale cohorts with greater numbers of people who consume these foods, or pooled analyses from multiple cohorts with varying levels of consumption. Methods such as diet optimisation modelling or linear modelling ${ }^{60}{ }^{61}$ could also be explored to identify optimal diets for disease prevention while respecting individual dietary choices.

\section{Strengths and limitations}

The strengths of this study include a large sample size, a long follow-up, and outcome ascertainment by linkage to medical records that minimised the loss to follow-up. Because the exposure of interest was distinct diet groups, defined by the exclusion of animal foods, the chance of misclassification of exposure was relatively low. Long term adherence to diet groups in the cohort was also generally high, and where possible we updated the exposure and important confounders at 14 year follow-up to allow for any changes. The analyses also included adjustment for multiple confounders, assessment of several possible mediators, and sensitivity analyses to confirm the robustness of the results.
Among the limitations of the present study, diet group was self reported, and reasons for choosing each diet were not recorded. Changes in diet group or other behaviours not captured by the follow-up were also possible, and the composition of vegetarian diets could have changed during follow-up owing to increasing availability of vegetarian foods, but differences in nutrient intakes between the diet groups were similar at baseline and at follow-up. ${ }^{96}$ Reverse causality is possible but not likely, because the results were similar after we excluded the first five years of follow-up, and most participants had followed their current diet (eg, vegetarian) for more than five years at the time of recruitment. Information on drug treatment use (including statins) at recruitment was not available. As with all observational studies, residual confounding from either dietary or non-dietary factors is possible, which might be particularly relevant if results were of borderline significance. Generalisability could be limited, because the present study was based predominantly on white European individuals.

\section{Conclusions}

Overall, the present study has shown that UK adults who were fish eaters or vegetarians had lower risks of ischaemic heart disease than meat eaters, but that vegetarians had higher risks of stroke. Future work should include further measurements of circulating levels of cholesterol subfractions, vitamin $\mathrm{B}_{12}$, amino acids, and fatty acids in the cohort to identify which factors might mediate the observed associations. Additional studies in other large scale cohorts with a high proportion of non-meat eaters are needed to confirm the generalisability of these results and assess their relevance for clinical practice and public health.

We thank all participants in the EPIC-Oxford cohort for their invaluable contribution.

Contributors: TYNT and TJK conceived and designed the research question. TYNT analysed the data and wrote the first draft of the manuscript; PNA created the figure and prepared the data for analysis; and PNA, KEB, AP-C, RCT, RC, and TJK provided input on data analysis and interpretation of results. All authors revised the manuscript critically for important intellectual content, and read and approved the final manuscript. TYNT is the guarantor. The corresponding author attests that all listed authors meet authorship criteria and that no others meeting the criteria have been omitted.

Funding: The work is supported by the UK Medical Research Council MR/M012190/1 and Wellcome Trust Our Planet Our Health (Livestock, Environment, and People, LEAP 205212/Z/16/Z). KEB is supported by the Girdlers' New Zealand Health Research Council Fellowship.

Competing interests: All authors have completed the ICMJE uniform disclosure form at www.icmje.org/coi_disclosure.pdf and declare: support from the UK Medical Research Council and Wellcome Trust for the submitted work; no financial relationships with any organisations that might have an interest in the submitted work in the previous three years; no other relationships or activities that could appear to have influenced the submitted work.

Ethical approval: The study protocol was approved by a multicentre research ethics committee (Scotland A research ethics committee), and all participants provided written informed consent.

Data sharing: The data access policy for the EPIC-Oxford study is available via the study website (www.epic-oxford.org/data-accesssharing-and-collaboration/).

The lead author (the manuscript's guarantor) affirms that this manuscript is an honest, accurate, and transparent account of the 
study being reported; that no important aspects of the study have been omitted; and that any discrepancies from the study as planned (and, if relevant, registered) have been explained.

This is an Open Access article distributed in accordance with the terms of the Creative Commons Attribution (CC BY 4.0) license, which permits others to distribute, remix, adapt and build upon this work, for commercial use, provided the original work is properly cited. See: http://creativecommons.org/licenses/by/4.0/.

1 Leahy E, Lyons S, Tol RS). An estimate of the number of vegetarians in the world. ESRI working paper 340. 2010. https://www.esri.ie/ publications/an-estimate-of-the-number-of-vegetarians-in-the-world/ 2 Bates B, Lennox A, Prentice A, et al. National Diet and Nutrition Survey: results from years 1,2,3,4 (combined) of the rolling programme (2008/2009-2011/2012). London; 2014. https://www. gov.uk/government/statistics/national-diet-and-nutrition-surveyresults-from-years-1-to-4-combined-of-the-rolling-programme-for2008-and-2009-to-2011-and-2012

3 The Vegan Society. Find out how many vegans are in Great Britain. 2016. https://www.vegansociety.com/whats-new/news/find-outhow-many-vegans-are-great-britain

4 Appleby PN, Key TJ. The long-term health of vegetarians and vegans. Proc Nutr Soc 2016;75:287-93. doi:10.1017/ S0029665115004334

5 Key TJ, Fraser GE, Thorogood M, et al. Mortality in vegetarians and nonvegetarians: detailed findings from a collaborative analysis of 5 prospective studies. Am J Clin Nutr 1999;70(Suppl):516S-24S. doi:10.1093/ajen/70.3.516s

6 Appleby PN, Crowe FL, Bradbury KE, Travis RC, Key TJ. Mortality in vegetarians and comparable nonvegetarians in the United Kingdom. Am / Clin Nutr 2016;103:218-30. doi:10.3945/ajcn.115.119461

7 Orlich MJ, Singh PN, Sabaté J, et al. Vegetarian dietary patterns and mortality in Adventist Health Study 2. JAMA Intern Med 2013;173:1230-8. doi:10.1001/jamainternmed.2013.6473

8 Crowe FL, Appleby PN, Travis RC, Key TJ. Risk of hospitalization or death from ischemic heart disease among British vegetarians and nonvegetarians: results from the EPIC-Oxford cohort study. Am I Clin Nutr 2013.97:597-603 doi:10.3945/ajen.112.044073

9 Davey GK, Spencer EA, Appleby PN, Allen NE, Knox KH, Key TJ. EPICOxford: lifestyle characteristics and nutrient intakes in a cohort of 33883 meat-eaters and 31546 non meat-eaters in the UK. Public Health Nutr 2003;6:259-69. doi:10.1079/PHN2002430

10 Appleby PN, Thorogood M, Mann JI, Key TJ. The Oxford Vegetarian Study: an overview. Am J Clin Nutr 1999;70(Suppl):525S-31S. doi:10.1093/ajcn/70.3.525s

11 Bingham SA, Gill C, Welch A, et al. Comparison of dietary assessment methods in nutritional epidemiology: weighed records $v .24 \mathrm{~h}$ recalls, food-frequency questionnaires and estimated-diet records. $\mathrm{Br} J$ Nutr 1994;72:619-43. doi:10.1079/BJN19940064

12 Bingham SA, Cassidy A, Cole TJ, et al. Validation of weighed records and other methods of dietary assessment using the 24 $\mathrm{h}$ urine nitrogen technique and other biological markers. $\mathrm{Br}$ J Nutr 1995;73:531-50. doi:10.1079/BJN19950057

13 Bingham SA, Gill C, Welch A, et al. Validation of dietary assessment methods in the UK arm of EPIC using weighed records, and 24-hour urinary nitrogen and potassium and serum vitamin $C$ and carotenoids as biomarkers. Int J Epidemiol 1997;26(Suppl 1):S137-51. doi:10.1093/ije/26.suppl 1.S137

14 Ministry of Agriculture Fisheries and Food. Food portion sizes. 2nd ed. Her Majesty's Stationary Office, 1993

15 Holland B, Welch A, Unwin I, et al. McCance and Widdowson's the composition of foods. 5th ed. Royal Society of Chemistry, 1991.

16 Townsend P. Poverty in the United Kingdom: a survey of household resources and standards of living. Allen Lane and Penguin Books, 1979

17 Wareham NJ, Jakes RW, Rennie KL, et al. Validity and repeatability of a simple index derived from the short physical activity questionnaire used in the European Prospective Investigation into Cancer and Nutrition (EPIC) study. Public Health Nutr 2003;6:407-13. doi:10.1079/PHN2002439

18 Spencer EA, Appleby PN, Davey GK, Key TJ. Validity of self-reported height and weight in 4808 EPIC-Oxford participants. Public Health Nutr 2002;5:561-5. doi:10.1079/PHN2001322

19 Bradbury KE, Crowe FL, Appleby PN, Schmidt JA, Travis RC, Key T). Serum concentrations of cholesterol, apolipoprotein A-I and apolipoprotein B in a total of 1694 meat-eaters, fish-eaters, vegetarians and vegans [correction in: Eur J Clin Nutr 2015;69:1180]. Eur / Clin Nutr 2014:68:178-83. doi:10.1038/ejcn.2013.248

20 Appleby PN, Davey GK, Key TJ. Hypertension and blood pressure among meat eaters, fish eaters, vegetarians and vegans in EPIC-Oxford. Public Health Nutr 2002:5:645-54. doi:10.1079/ PHN2002332

21 Tong TY, Key TJ, Sobiecki JG, Bradbury KE. Anthropometric and physiologic characteristics in white and British Indian vegetarians and nonvegetarians in the UK Biobank. Am J Clin Nutr 2018;107:909-20. doi:10.1093/ajcn/nqy042

22 Chiu Y-FF, Hsu CC, Chiu THTT, et al. Cross-sectional and longitudinal comparisons of metabolic profiles between vegetarian and non-vegetarian subjects: a matched cohort study. $\mathrm{Br}$ J Nutr 2015:114:1313-20, doi:10.1017/S0007114515002937

23 Tonstad S, Stewart K, Oda K, Batech M, Herring RP, Fraser GE. Vegetarian diets and incidence of diabetes in the Adventist Health Study-2. Nutr Metab Cardiovasc Dis 2013;23:292-9. doi:10.1016/j. numecd.2011.07.004

24 Forouzanfar MH, Alexander L, Anderson HR, et al, GBD 2013 Risk Factors Collaborators. Global, regional, and national comparative risk assessment of 79 behavioural, environmental and occupational, and metabolic risks or clusters of risks in 188 countries, 19902013: a systematic analysis for the Global Burden of Disease Study 2013. Lancet 2015;386:2287-323. doi:10.1016/S01406736(15)00128-2

25 Easton DF, Peto J, Babiker AGAG. Floating absolute risk: an alternative to relative risk in survival and case-control analysis avoiding an arbitrary reference group. Stat Med 1991;10:1025-35. doi:10.1002/ sim.4780100703

26 Lewington S, Whitlock G, Clarke R, et al, Prospective Studies Collaboration. Blood cholesterol and vascular mortality by age, sex, and blood pressure: a meta-analysis of individual data from 61 prospective studies with 55,000 vascular deaths. Lancet 2007;370:1829-39. doi:10.1016/S0140-6736(07) 61778-4

27 Chang-Claude J, Hermann S, Eilber U, Steindorf K. Lifestyle determinants and mortality in German vegetarians and healthconscious persons: results of a 21-year follow-up. Cancer Epidemiol Biomarkers Prev 2005;14:963-8. doi:10.1158/1055-9965.EPI-040696

28 Dinu M, Abbate R, Gensini GF, Casini A, Sofi F. Vegetarian, vegan diets and multiple health outcomes: A systematic review with meta-analysis of observational studies. Crit Rev Food Sci Nutr 2017:57:3640-9. doi:10.1080/10408398.2016.1138447

29 Collins R, Reith C, Emberson J, et al. Interpretation of the evidence for the efficacy and safety of statin therapy. Lancet 2016;388:2532-61. doi:10.1016/S0140-6736(16)31357-5

30 Ference BA, Yoo W, Alesh I, et al. Effect of long-term exposure to lower low-density lipoprotein cholesterol beginning early in life on the risk of coronary heart disease: a Mendelian randomization analysis. J Am Coll Cardiol 2012;60:2631-9. doi:10.1016/j. jacc.2012.09.017

31 Hindy G, Engström G, Larsson SC, et al, Stroke Genetics Network (SiGN). Role of blood lipids in the development of ischemic stroke and its subtypes: a mendelian randomization study. Stroke 2018;49:820-7. doi:10.1161/STROKEAHA.117.019653

32 Di Angelantonio E, Sarwar N, Perry P, et al, Emerging Risk Factors Collaboration. Major lipids, apolipoproteins, and risk of vascular disease. JAMA 2009;302:1993-2000. doi:10.1001/ jama.2009.1619

33 Baigent C, Blackwell L, Emberson J, et al, Cholesterol Treatment Trialists' (CTT) Collaboration. Efficacy and safety of more intensive lowering of LDL cholesterol: a meta-analysis of data from 170,000 participants in 26 randomised trials. Lancet 2010;376:1670-81. doi:10.1016/S0140-6736(10)61350-5

34 Thorogood M, Carter R, Benfield L, McPherson K, Mann JI. Plasma lipids and lipoprotein cholesterol concentrations in people with different diets in Britain. Br Med I (Clin Res Ed) 1987;295:351-3. doi:10.1136/bmj.295.6594.351

35 Aune D, Giovannucci E, Boffetta P, et al. Fruit and vegetable intake and the risk of cardiovascular disease, total cancer and all-cause mortality-a systematic review and dose-response meta-analysis of prospective studies. Int J Epidemiol 2017;46:1029-56. doi:10.1093/ije/dyw319

36 Afshin A, Micha R, Khatibzadeh S, Mozaffarian D. Consumption of nuts and legumes and risk of incident ischemic heart disease, stroke, and diabetes: a systematic review and meta-analysis. Am / Clin Nutr 2014;100:278-88. doi:10.3945/ajcn.113.076901

37 Crowe FL, Key TJ, Appleby PN, et al. Dietary fibre intake and ischaemic heart disease mortality: the European Prospective Investigation into Cancer and Nutrition-Heart study. Eur J Clin Nutr 2012;66:950-6. doi:10.1038/ejcn.2012.51

38 Amarenco P, Bogousslavsky J, Callahan A3rd, et al, Stroke Prevention by Aggressive Reduction in Cholesterol Levels (SPARCL) Investigators. High-dose atorvastatin after stroke or transient ischemic attack. $N$ Engl J Med 2006;355:549-59. doi:10.1056/NEJMoa061894

39 Iso H, Jacobs DRJr, Wentworth D, Neaton JD, Cohen JD. Serum cholesterol levels and six-year mortality from stroke in 350,977 men screened for the multiple risk factor intervention trial. $N$ Engl Med 1989;320:904-10. doi:10.1056/NEJM198904063201405

40 Yano K, Reed DM, MacLean Cl. Serum cholesterol and hemorrhagic stroke in the Honolulu Heart Program. Stroke 1989;20:1460-5. doi:10.1161/01.STR.20.11.1460 
41 Wang X, Dong Y, Qi X, Huang C, Hou L. Cholesterol levels and risk of hemorrhagic stroke: a systematic review and meta-analysis. Stroke 2013;44:1833-9. doi:10.1161/STROKEAHA.113.001326

42 Sun L, Clarke R, Bennett D, et al, China Kadoorie Biobank Collaborative Group, International Steering Committee, International Co-ordinating Centre Oxford, National Co-ordinating Centre, Beijing, Regional Co-ordinating Centres. Causal associations of blood lipids with risk of ischemic stroke and intracerebral hemorrhage in Chinese adults. Nat Med 2019;25:569-74. doi:10.1038/s41591-0190366-X

43 Kinjo Y, Beral V, Akiba S, et al. Possible protective effect of milk, meat and fish for cerebrovascular disease mortality in Japan. $J$ Epidemiol 1999;9:268-74. doi:10.2188/jea.9.268

44 Sauvaget C, Nagano J, Allen N, Grant EJ, Beral V. Intake of animal products and stroke mortality in the Hiroshima/Nagasaki Life Span Study Int J Epidemiol 2003.32:536-43 doi:10.1093/ije/dyg151

45 Takeya Y, Popper JS, Shimizu Y, Kato H, Rhoads GG, Kagan A. Epidemiologic studies of coronary heart disease and stroke in Japanese men living in Japan, Hawaii and California: incidence of stroke in Japan and Hawaii. Stroke 1984;15:15-23. doi:10.1161/01. STR.15.1.15

46 Sauvaget C, Nagano J, Hayashi M, Yamada M. Animal protein, animal fat, and cholesterol intakes and risk of cerebral infarction mortality in the adult health study. Stroke 2004;35:1531-7. doi:10.1161/01. STR.0000130426.52064.09

47 Gilsing AMJ, Crowe FL, Lloyd-Wright Z, et al. Serum concentrations of vitamin B12 and folate in British male omnivores, vegetarian and vegans: results from a cross-sectional analysis of the EPICOxford cohort study. Eur J Clin Nutr 2010;64:933-9. doi:10.1038/ ejcn.2010.142

48 Crowe FL, Steur M, Allen NE, Appleby PN, Travis RC, Key TJ. Plasma concentrations of 25-hydroxyvitamin D in meat eaters, fish eaters, vegetarians and vegans: results from the EPIC-Oxford study. Public Health Nutr 2011;14:340-6. doi:10.1017/S1368980010002454

49 Schmidt JA, Rinaldi S, Scalbert A, et al. Plasma concentrations and intakes of amino acids in male meat-eaters, fish-eaters, vegetarians and vegans: a cross-sectional analysis in the EPIC-Oxford cohort. Eur J Clin Nutr 2016;70:306-12. doi:10.1038/ejcn.2015.144

50 Rosell MS, Lloyd-Wright Z, Appleby PN, Sanders TA, Allen NE, Key TJ. Long-chain n-3 polyunsaturated fatty acids in plasma in British meateating, vegetarian, and vegan men. Am J Clin Nutr 2005;82:327-34. doi:10.1093/ajcn/82.2.327

51 Dong H, Pi F, Ding Z, et al. Efficacy of supplementation with B vitamins for stroke prevention: a network meta-analysis of randomized controlled trials. PLoS One 2015;10:e0137533. doi:10.1371/ journal.pone.0137533

52 Iso H, Stampfer MJ, Manson JE, et al. Prospective study of fat and protein intake and risk of intraparenchymal hemorrhage in women. Circulation 2001;103:856-63. doi:10.1161/01.CIR.103.6.856
53 Sacks FM, Lichtenstein AH, Wu JHY, et al, American Heart Association. Dietary fats and cardiovascular disease: a presidential advisory from the American Heart Association [correction in: Circulation 2017;136:e195]. Circulation 2017;136:e1-23.

54 McGreevy C, Williams D. New insights about vitamin D and cardiovascular disease: a narrative review. Ann Intern Med 2011;155:820-6. doi:10.7326/0003-4819-155-12201112200-00004

55 Rosendorff C, Lackland DT, Allison M, et al, American Heart Association, American College of Cardiology, and American Society of Hypertension. Treatment of hypertension in patients with coronary artery disease: a scientific statement from the American Heart Association, American College of Cardiology, and American Society of Hypertension. Circulation 2015;131:e435-70. doi:10.1161/ CIR.0000000000000207

56 Lacey B, Lewington S, Clarke R, et al, China Kadoorie Biobank collaborative group. Age-specific association between blood pressure and vascular and non-vascular chronic diseases in 0.5 million adults in China: a prospective cohort study. Lancet Glob Health 2018;6:e641-9. doi:10.1016/S2214-109X(18)30217-1

57 Tong TYN, Appleby PN, Bradbury KE, Key TJ. Cross-sectional analyses of participation in cancer screening and use of hormone replacement therapy and medications in meat eaters and vegetarians: the EPIC-Oxford study. BMJ Open 2017;7:e018245. doi:10.1136/ bmjopen-2017-018245

58 Sinha S, Myint PK, Luben RN, Khaw K-T. Accuracy of death certification and hospital record linkage for identification of incident stroke. BMC Med Res Methodol 2008;8:74. doi:10.1186/1471-2288-8-74

59 Wright FL, Green J, Canoy D, Cairns BJ, Balkwill A, Beral V, Million Women Study Collaborators. Vascular disease in women: comparison of diagnoses in hospital episode statistics and general practice records in England. BMC Med Res Methodol 2012;12:161. doi:10.1186/1471-2288-12-161

60 Wilson N, Nghiem N, Ni Mhurchu C, Eyles H, Baker MG, Blakely T. Foods and dietary patterns that are healthy, low-cost, and environmentally sustainable: a case study of optimization modeling for New Zealand. PLoS One 2013;8:e59648. doi:10.1371/journal. pone.0059648

61 Masset G, Monsivais P, Maillot M, Darmon N, Drewnowski A. Diet optimization methods can help translate dietary guidelines into a cancer prevention food plan. J Nutr 2009;139:1541-8. doi:10.3945/ jn.109.104398

62 Sobiecki JG, Appleby PN, Bradbury KE, Key TJ. High compliance with dietary recommendations in a cohort of meat eaters, fish eaters, vegetarians, and vegans: results from the European Prospective Investigation into Cancer and Nutrition-Oxford study. Nutr Res 2016:36:464-77. doi:10.1016/i.nutres.2015.12.016

Web appendix: Supplementary material 